\title{
Research Paper \\ Effects of High-intensity Interval Training on Body Composition and Hormone Growth Agents in Overweight Adolescent Boys
}

\author{
Javad Vakili ${ }^{1}(1)$, Vahid Sari Sarraf ${ }^{1}$ (1), *Towhid Khanvari ${ }^{1}$ (])
}

1. Department of Exercise Physiology, Faculty of physical Education and Sports Science, University of Tabriz, Tabriz, Iran.

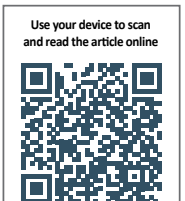

Citation: Vakili J, Sari Sarraf V, Khanvari T. [The Effect of Two Types of High Intensity Interval Training for 8 Weeks on Body Composition and Hormone Growth Agents in Overweight Adolescent Boys (Persian)]. Journal of Arak University of Medical Sciences (JAMS). 2021; 24(1):136-149. https://doi.org/10.32598/JAMS.24.1.3619.2

doi ${ }^{\prime} h t$ tps://doi.org/10.32598/JAMS.24.1.3619.2

Keywords:

High-Intensity interval training, Overweight, Insulin-Like growth factor I, Growth hormone

\section{ABSTRACT}

Background and Aim Obesity is a clinical condition that affects the growth-related hormonal system, especially in adolescents. High-Intensity Intermittent Training (HIIT) might reduce such destructive effects. Methods \& Materials In this quasi-experimental research, among adolescent boys [age: $13-18$ years, height: $154 \pm 8 \mathrm{~cm}$, Body Mass Index (BMI): $27.05 \pm 1.4 \mathrm{~kg} / \mathrm{m}^{2}$ ], 30 volunteer subjects were selected. The study participants were divided into 3 groups of short-term and long-term periodic exercises as well as controls. The short-term exercise group performed 9 bouts of 30 seconds with 150-second breaks between sessions; the long-term exercise group performed 4 bouts of 150 seconds with 240-second breaks between sessions 3 times a week for 8 weeks. Body composition and blood samples were obtained 48 hours before and after conducting the training protocol to measure the desired parameters. The changes of each index were analyzed by repeated-measures Analysis of Variance (ANOVA) at the significant level of $P<0.05$.

Ethical Considerations This study was approved by the Ethics Committee of Tabriz University (Code: IR.TABRIZU.REC.1398.021).

Results After 8 weeks of HIIT, growth hormone increased and thigh, arm circumference, and fat percentages decreased $(P<0.05)$. However, no changes were detected in the insulin-like growth factor I, BMI, and weight of the examined subjects ( $P>0.05)$.

Conclusion Short- and long-term HIIT have the potential to improve the growth hormone status and body composition of overweight adolescents.

\section{Extended Abstract}

\section{Introduction}

besity is among the main public health challenges of the $21^{\text {st }}$ century [1]; it has reached epidemic proportions in Western countries [2]. This condition significantly impacts the development of other metabolic diseases, especially in adolescents. Besides, obesity is associated with de- creased GH secretion [16] as well as serum IGF-I [17]. Furthermore, High-Intensity Intermittent Training (HIIT), in different models, usually presents different effects on growth hormone concentrations $[29,30]$ as well as IGF-1 [34]. It is necessary to explore approaches to improve the effects of obesity and overweight in adolescents; thus, this study aimed to compare the effects of two types of HIIT for 8 weeks on body composition and resting levels of some anabolic factors in overweight adolescent boys.

* Corresponding Author:

Towhid Khanvari

Address: Department of Exercise Physiology, Faculty of physical Education and Sports Science, University of Tabriz, Tabriz, Iran.

Tel: +98 (41) 42225191

E-mail:milad_felps@yahoo.com 
Table 1. The values of physical and hormonal components before intervention in different research groups

\begin{tabular}{|c|c|c|c|c|c|}
\hline \multirow{2}{*}{\multicolumn{2}{|c|}{ Indicators / Groups }} & \multicolumn{3}{|c|}{ Mean士SD } & \multirow{2}{*}{$\begin{array}{l}\text { The Significance Level } \\
\text { of the Shapirovilk Test }\end{array}$} \\
\hline & & \multirow{2}{*}{$\begin{array}{c}\text { HIIT (Short-term) } \\
14.88 \pm 1.65\end{array}$} & \multirow{2}{*}{$\begin{array}{c}\text { HIIT (Long-term) } \\
14.88 \pm 1.26\end{array}$} & \multirow{2}{*}{$\begin{array}{c}\text { Control } \\
14.33 \pm 1.58\end{array}$} & \\
\hline Age (y) & & & & & - \\
\hline Height $(\mathrm{cm})$ & & $151.98 \pm 9$ & $156.12 \pm 8$ & $156.23 \pm 10$ & - \\
\hline \multirow{2}{*}{ Weight (kg) } & Pre-test & $60.47 \pm 3.26$ & $65.66 \pm 4.90$ & $67.7 \pm 4.736$ & \multirow{2}{*}{0.329} \\
\hline & Post-test & $58.84 \pm 4.21$ & $64.80 \pm 3.88$ & $67.200 \pm 5.17$ & \\
\hline \multirow[b]{2}{*}{ Fat percentage } & Pre-test & $26.58 \pm 2.22^{*}$ & $27.55 \pm 2.06 *$ & $27.30 \pm 2.74$ & \multirow[b]{2}{*}{0.096} \\
\hline & Post-test & $24.36 \pm 1.54$ & $25.22 \pm 2.00$ & $27.12 \pm 2.36$ & \\
\hline \multirow{2}{*}{$\mathrm{BMI}\left(\mathrm{kg} / \mathrm{m}^{2}\right)$} & Pre-test & $27.14 \pm 1.21$ & $26.72 \pm 1.39$ & $27.30 \pm 2.18$ & \multirow[b]{2}{*}{0.912} \\
\hline & Post-test & $26.35 \pm 1.53$ & $26.38 \pm 1.03$ & $26.60 \pm 1.31$ & \\
\hline \multirow[b]{2}{*}{ Waist to hip ratio } & Pre-test & 1.026 & 1.029 & 1.043 & \multirow[b]{2}{*}{0.852} \\
\hline & Post-test & 1.03 & 1.029 & 1.049 & \\
\hline \multirow{2}{*}{ Round thigh } & Pre-test & $47.88 \pm 5.57 *$ & $47.33 \pm 4.00$ & $56.88 \pm 11.03$ & \multirow{2}{*}{0.075} \\
\hline & Post-test & $45.94 \pm 5.38$ & $47.33 \pm 4.87$ & $53.55 \pm 5.89$ & \\
\hline \multirow[b]{2}{*}{ Arm circumference } & Pre-test & $27.77 \pm 1.85 *$ & $28.88 \pm 3.01^{*}$ & $29.81 \pm 3.55$ & \multirow[b]{2}{*}{0.097} \\
\hline & Post-test & $25.34 \pm 2.24$ & $25.79 \pm 2.75$ & $31.44 \pm 3.24$ & \\
\hline \multirow[b]{2}{*}{$\mathrm{Vo}_{2} \max (\mathrm{ml} / \mathrm{kg} / \mathrm{min})$} & Pre-test & $33.08 \pm 1.85 *$ & $32.72 \pm 1.51^{*}$ & $31.65 \pm 2.39$ & \multirow[b]{2}{*}{0.986} \\
\hline & Post-test & $38.30 \pm 1.51$ & $36.70 \pm 1.99$ & $32.08 \pm 2.17$ & \\
\hline \multirow{2}{*}{ Growth hormone ( $\mu \mathrm{g} / \mathrm{l})$} & Pre-test & $0.30 \pm 0.03$ & $0.26 \pm 0.03$ & $0.28 \pm 0.05$ & \multirow{2}{*}{0.638} \\
\hline & Post-test & $0.35 \pm 0.04 *$ & $0.34 \pm 0.04 *$ & $0.28 \pm 0.04$ & \\
\hline \multirow{2}{*}{ IGF-1 ( $\mu \mathrm{g} / \mathrm{I})$} & Pre-test & $165.66 \pm 13.07$ & $159.22 \pm 10.05$ & $168.44 \pm 10.42$ & \multirow{2}{*}{0.081} \\
\hline & Post-test & $174.55 \pm 8.64$ & $164.88 \pm 7.59$ & $168.55 \pm 9.59$ & \\
\hline
\end{tabular}

*Intragroup changes; "Intergroup changes.

\section{Materials and Methods}

This was a quasi-experimental study with a pre-test, posttest and a control group design. After inviting to participate in the study, initial assessments, including height, weight, body fat percentage, waist circumference, hip circumference, and other anthropometric indices were performed. Of the eligible and volunteer individuals, 30 subjects were selected as the study sample. The study subjects provided informed consent forms., Accordingly the research subjects underwent medical examinations. After measuring body composition [height, weight, Body Mass Index (BMI), body fat percentage, and waist circumference] and blood parameters, the examined individuals were divided into 3 groups of
10 subjects, as follows: short-term training (nine 30-second intervals with 150 -second breaks between sessions), longterm intermittent training (four 150-second intervals with 240-second breaks), and controls who were trained for 8 weeks. Body composition and blood samples were obtained 48 hours before and after performing the training protocol to measure the relevant indicators. The changes of each index were analyzed by repeated-measures Analysis of Variance (ANOVA) at a significant level of $\mathrm{P}<0.05$.

\section{Results}

The results of repeated-measures ANOVA suggested that short-term and long-term HIIT training presented optimal 
and significant effects on body fat percentage, hip circumference, and arm circumference; however, this exercise did not change BMI, weight, waist, and hips circumferences in the study subjects. Moreover, the provided short-term and long-term HIIT training increased serum growth hormone levels in the examined overweight adolescents and indicated no effect on IGF-1 levels. Table 1 lists all Pre-test and Post-test data related to the studied indicators.

\section{Discussion and Conclusion}

Consistent with the present study findings, Abdul Rahman et al. (2018) reported that 7 weeks of HIIT training o increased GH in men. They declared that one of the reasons for the increase in GH induced by HIIT exercises is elevated catecholamines. Increased epinephrine and norepinephrine following strenuous activity can enhance GH production and release by affecting anabolic pathways [31]. Moreover, Stekus et al. (2002) argued that the duration of exercise is effective in increasing the GH. In their study, a 30 -second HIIT episode further increased serum GH than a 6-second HIIT. The researchers attributed the difference to the high level of work performed in the 30 -second activity [30]. However, inconsistent with the present study data and in one of the few studies, Hejazi (2017) examined the effect of 10 weeks of short-term HIIT training; they concluded this type of training can significantly increase IGF-1 [34]. The exercise conducted in Hejazi's study was short-term; thus, the longer training period in Hejazi study may have caused this difference. In the present study, short-term HIIT training increased IGF-1 values by 10 units despite not being significant.

\section{Ethical Considerations}

\section{Compliance with ethical guidelines}

This study was approved by the Ethics Committee of Tabriz University (Code: IR.TABRIZU.REC.1398.021).

\section{Funding}

This study was extracted from the $\mathrm{PhD}$. dissertation of third author at the Department of Exercise Physiology, Faculty of physical Education and Sports Science, University of Tabriz, Tabriz.

\section{Authors' contributions}

Conceptualization, text writing and review: All authors; Research and sampling method: Tohid Khanvari; Data analysis: Tohid Khanvari, Javad Vakili.

\section{Conflicts of interest}

The authors declared no conflict of interest. 


\title{
تأثير دو نوع تمرين تناوبى با شدث بالا به مدت هشت هفته بر تركيب بدنى و عوامل هورمونى

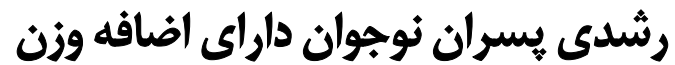

\author{
جواد وكيلى' ه. وحيد سارى صراف' ـ. "توحيد خانوارى' (ه)
}

ا. كروه فيزيولورثى ورزشى، دانشكده تربيت بدنى و علوم ورزشى، دانشعاه تبريز، تبريز، ايران.

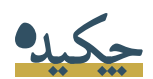

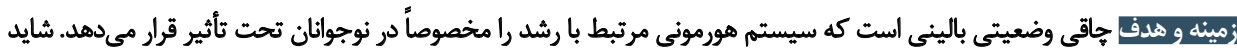

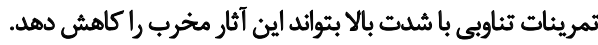

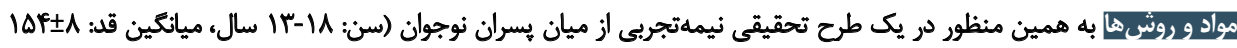

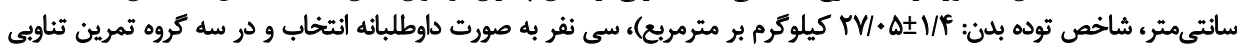

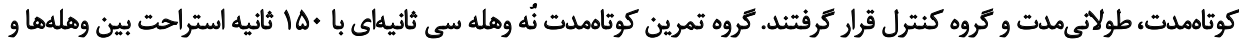

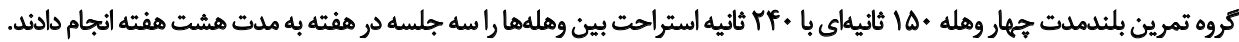

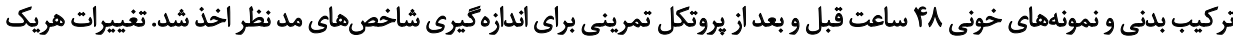

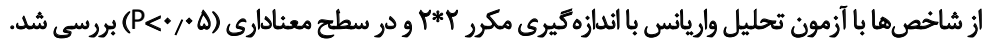

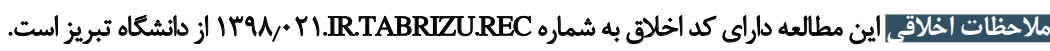

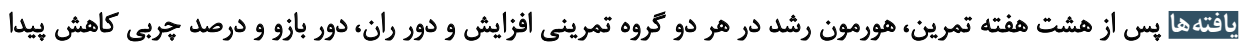

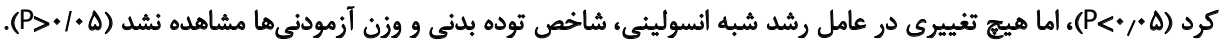

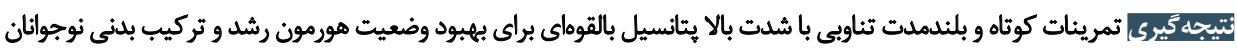
داراي اضافه وزن مارد.

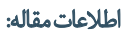

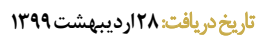

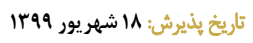

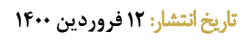

كليدوأوها: تمرينات تناوبى با شدات بالا، اضافه وزن، عامل تئل روشد شبه ائسوليني، وزني هورمون رشد شيه
\end{abstract}

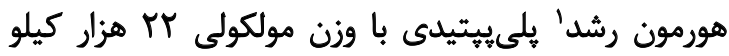

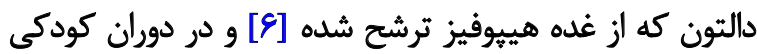

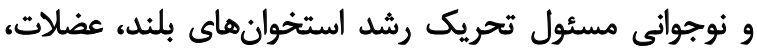

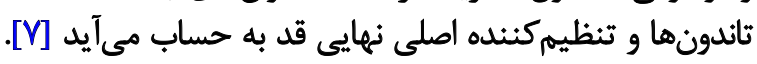

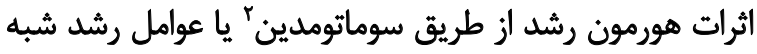

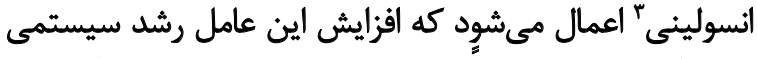

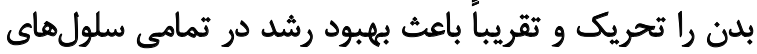

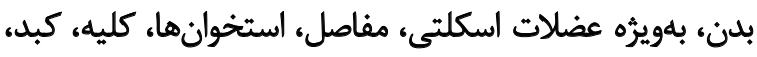

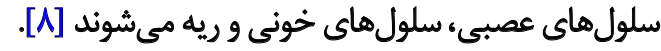

1. Growth Hormone (GH)

2. Somatomedins

3. Insulin-like Growth Factors (IGFs)

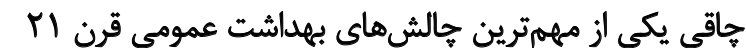

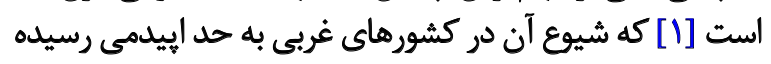

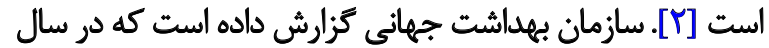

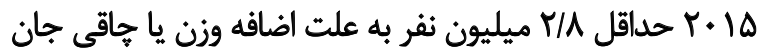

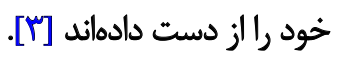

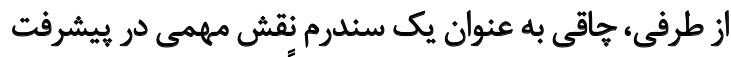

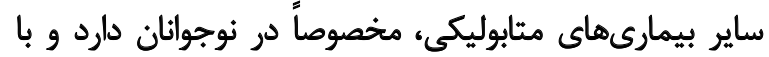

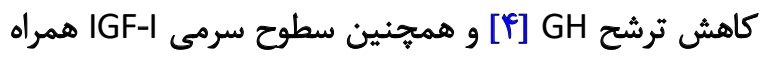

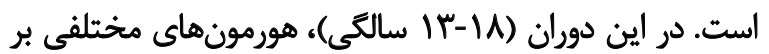

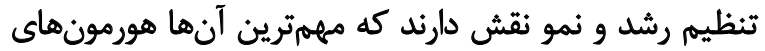

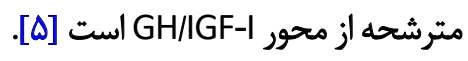


با نود ثانيه استراحت غيرفعال از يكديكر جدا مي دشدند) نتوانست

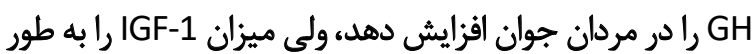

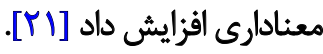

ازاينرو،تأثير انواع مختلفى ازتمرينات تناوبى باشدتابتالابر محور GH/IGF-1

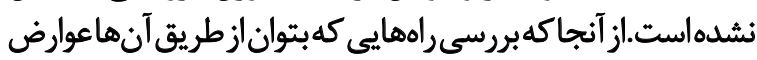

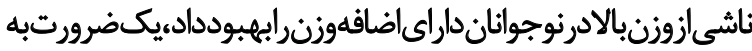

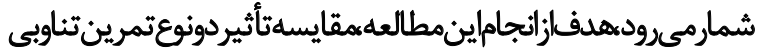

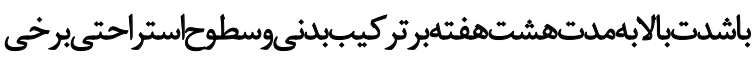
عوامل آنابوليك در يسران نوجوان داراى اضافه وزن است.

\section{مواد ورش ها}

روش تحقيق حاضر از نوع نيملتجربى با طرح ييشآزمون

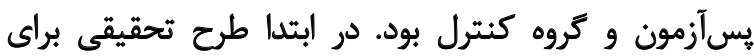

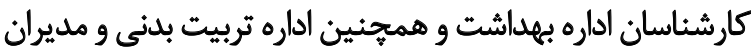

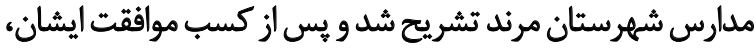

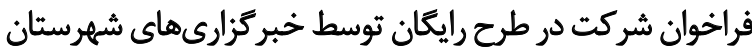

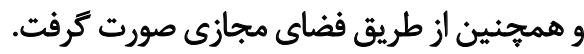

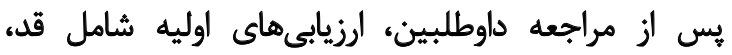

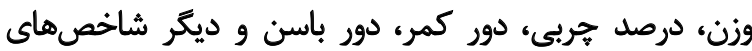

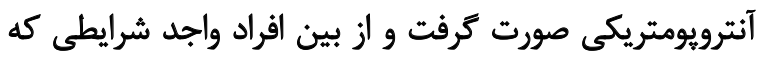

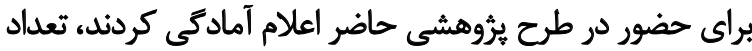
سى نفر به عنوان نمونه انتخاب شدرند حاضد اعلام

شاخصهاى ورود به تحقيق شامل سن (Tا Iا تا IM سال)،

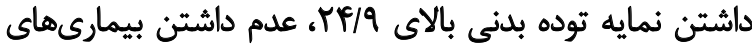

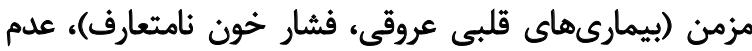

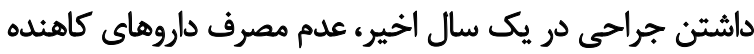

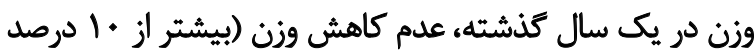

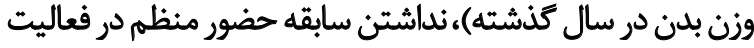

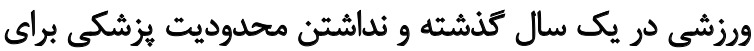

$$
\text { مشاركت در فعاليت بود. }
$$

يس از تكميل فرم رضايت آكاهانه توسط نوجوانان و والدين

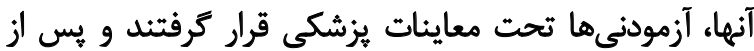

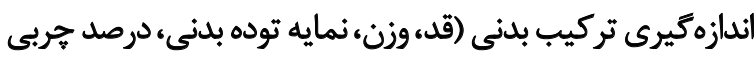

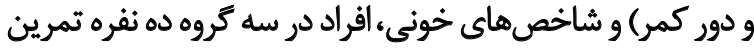
تناوبى كوتاهمدت، تمرين تناوبى بلندمدت و وركان

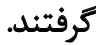

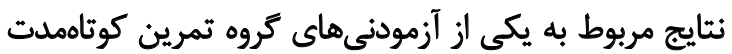

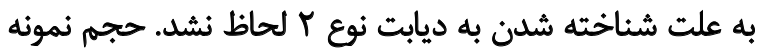

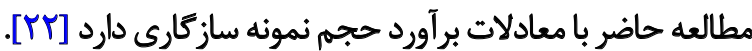

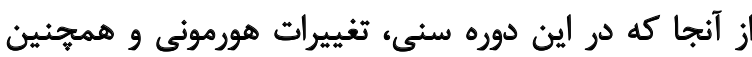

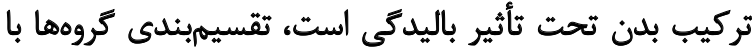

تواقى با كاهش GH و GF-1 اثرات مفيد اين دو هورمون

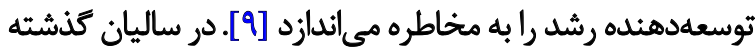

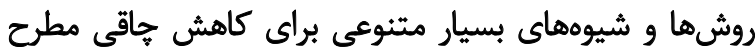

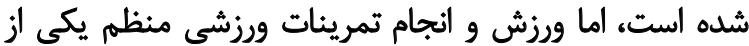

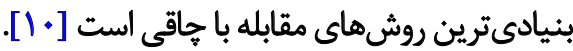
على غعم محرز شدن مزاياي تمرينات ورزشى، بسيارى از افراد

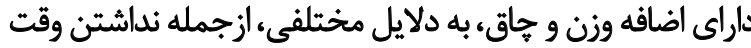

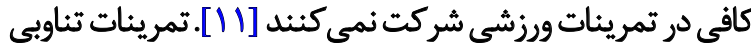

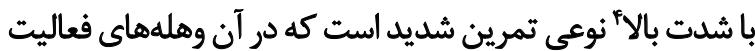

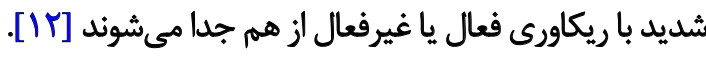
اين تمرينات اكسيداسيون جربي و كربوهيدرات را در عضله

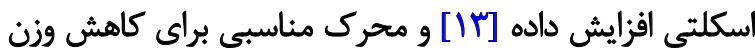

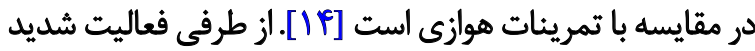

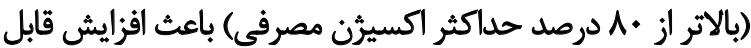

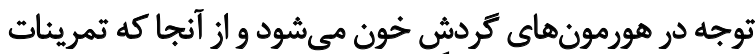

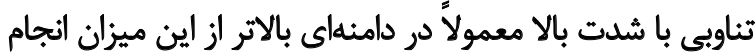

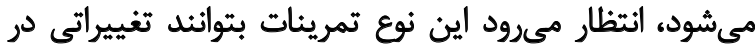

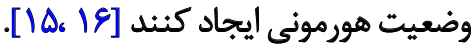

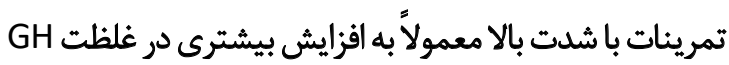

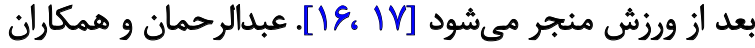

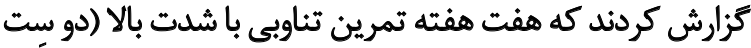

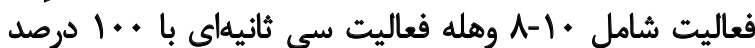

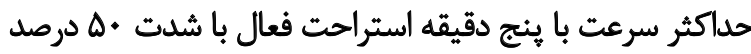

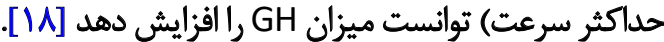

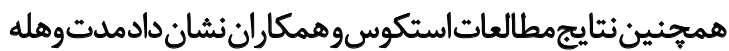

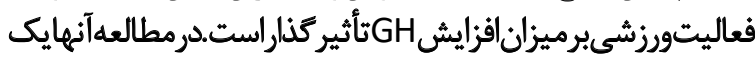

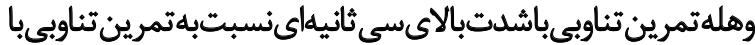

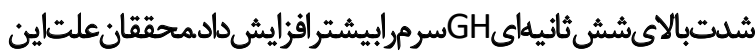

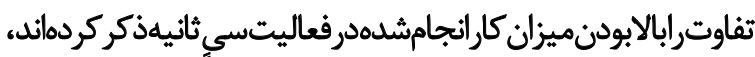

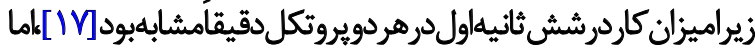

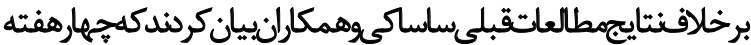

تمرين تناوبى با شدت بالا بر ميزان GH تأثيرى ندارد [19 19]. همجنين سورى و همكاران در مطالعه روى مردان داراى اضافه بانه

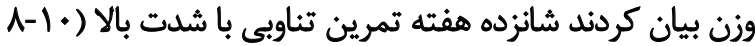

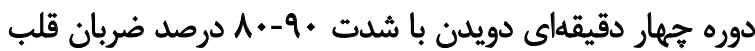

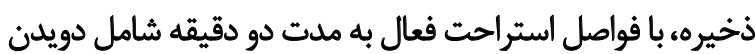

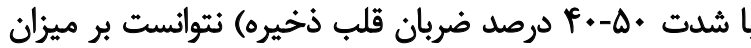

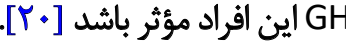

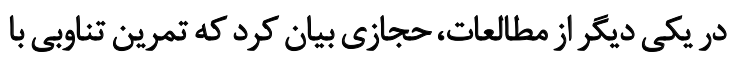

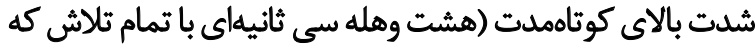

4. High Intensity Interval Training 


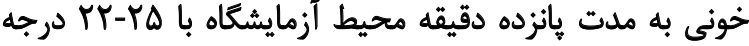

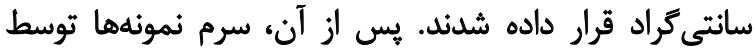

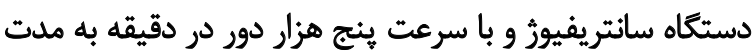

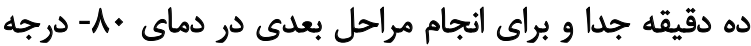
سانتى كراد قرار داده شد.

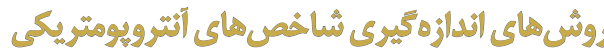

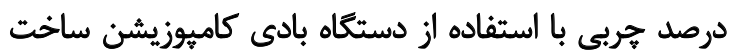

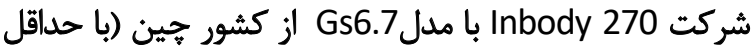

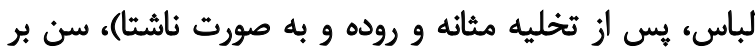

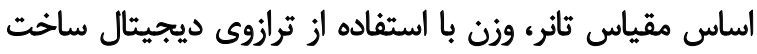

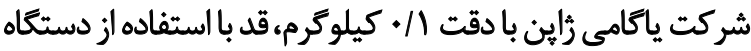

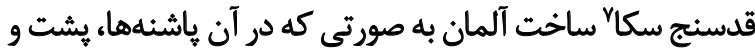

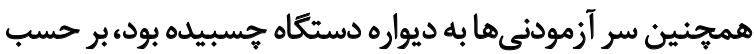

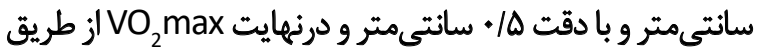

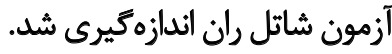

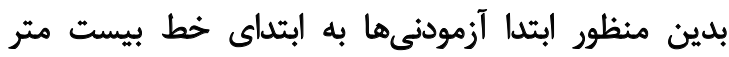

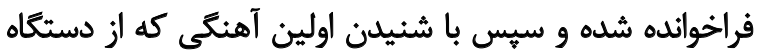

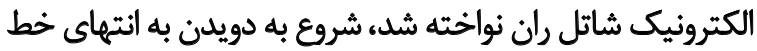

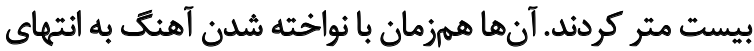
بيست متر رسيده و دوباره به نقطه شروع باز بشتشتند. در دقيقه اول آزمون، اين رفت و بركشتها با سرعت هشت

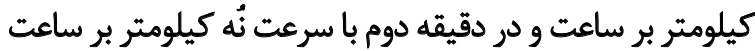

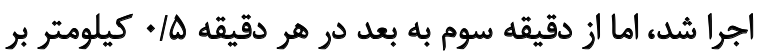

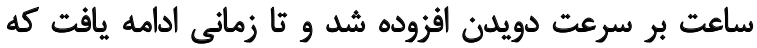

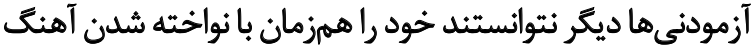

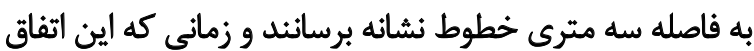

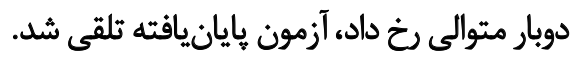
همجنين، آزمودنىها يك هفته قبل از شروع يروتكل با تكميل

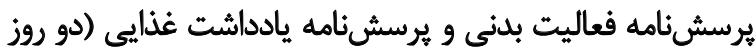

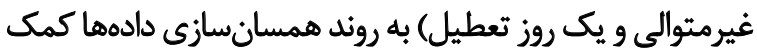

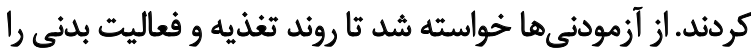
تا انتهاى مطالعه بدون تغيير ادامه دهند.

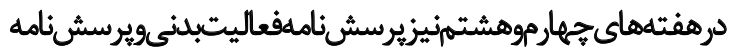

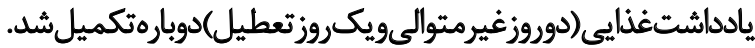

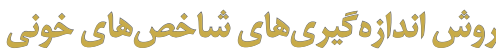

به دليل انجام فعاليت با شدت بالا و احتمال تغييرات حجم

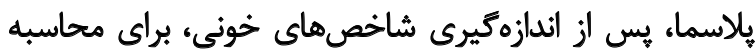
تغييرات حجم يلاسما و ضريب اصلاحشده مقادير، از معادله بانه
استفاده از مقياس تعيين مراحل بلوغ تائره و توسط متخصص بوري

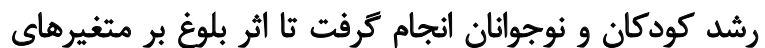

$$
\text { وابسته به حداقل برسد. }
$$

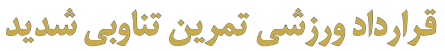

همه آزمودنى ها با هدف آشناسازى و افزايش آمادتى اوليه در

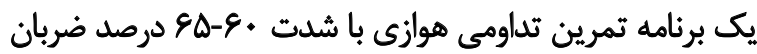

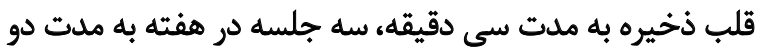

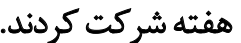

سيس كروه تمرين كوثامدت و بلندمدت به مدت هشت هفته

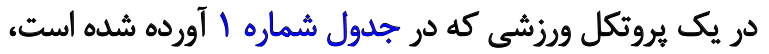

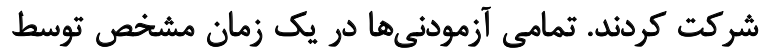

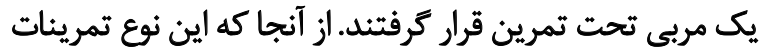

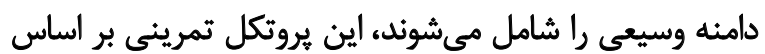

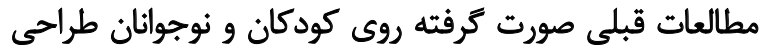

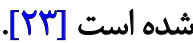

هر جلسه برنامه تمرينى با ده الى يانزده دقيقه كرم كردن

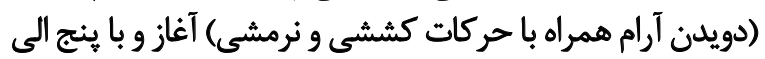

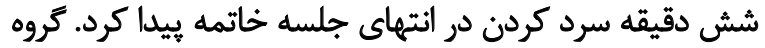

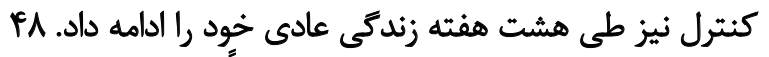

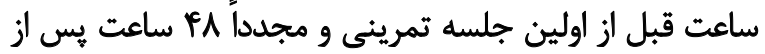

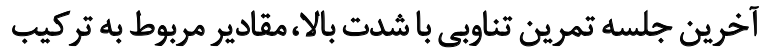

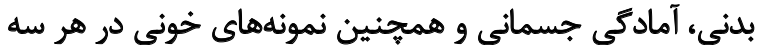

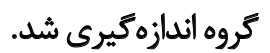

رئيم غذايى روزانه آزمودنىها قبل از شروع مطالعه و در بر بردي

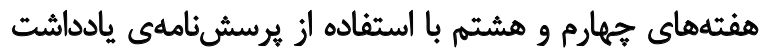
تغذيهاى سه روزه كنترل شد. همه آزمودنى هانها هنغام تمرينا

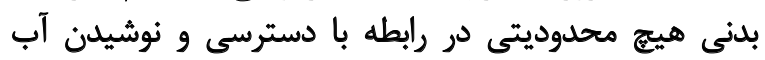
نباشتئل

ياقتهها

تمام آزمودنىها يك روز قبل و يك روز يُ يس از اولين جلسه

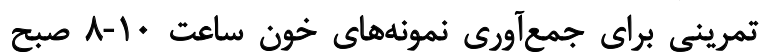

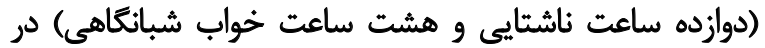
آزمايشكاه حاضر شدند.

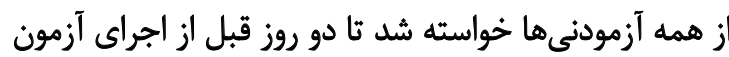

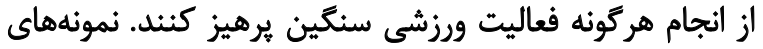

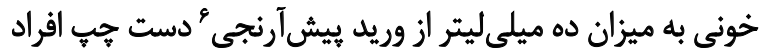

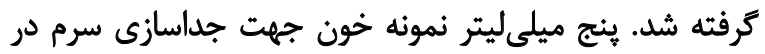
لوله آزمايش مخصوص ريخته شد. براى تشكيل لخته، نمونه جناي

5. Tanner Stages of Puberty

6. Antecubital Vein 
جدول ا. يروتكل ورزشى تدوينشده در ئوهش جارى

\begin{tabular}{|c|c|c|c|c|}
\hline ينجمه تا هشتم & هفته سوم و جهارم & هفته اول و دوم & جارامتر & كروه تمرينى \\
\hline ir & 9 & 8 & تعداد وهلهها & \\
\hline r. & $r$ & Fo & مدت كار (ثانيه) & \\
\hline 10. & 10. & 10. & هلت استراحت (ثانيه) & \\
\hline غير فعال & ع غِيرفَعال & ع عيرفعال & نوع استراحت & كوتاهملت \\
\hline •11- . إدرصد حلاكثر سرعت & "11-. .1 درصد حلاكثر سرعت & "11-. الرهل حداكثر سرعت & كلت كار & \\
\hline$\pi / \Delta$ & re/o & iv & ملت تمرين (دقيقه) & \\
\hline 8 & $f$ & r & تعداد وهلهها & \\
\hline 10. & 10. & 10. & مدت كار (ثانيه) & \\
\hline r. & rif. & $r v$ & ملت استراحت (ثانيه) & \\
\hline هو-+9 درصد حداكثر سرعت & هو-،9 درصد حداكثر سرعت & هو-•ج درصد حداكثر سرعت & 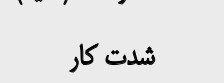 & بلنلمدت \\
\hline شدت QFا درصد حداكثر سرعت & شدت هز درصد حداكثر سرعت & شدت هأ درصد حداكثر سرعت & نوع استراحت & \\
\hline re & re & $1 \& / 0$ & ملت تمرين (دقيقه) & \\
\hline
\end{tabular}

مورد مطالعه تفاوت معنادارى با يكديكَر ندارند. نتايج تحليل واريانس در اندازههاي مكرر نيز نشان داد كه

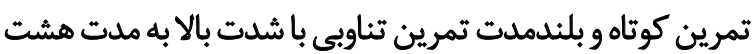

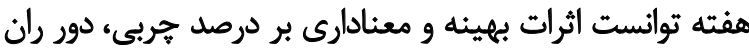

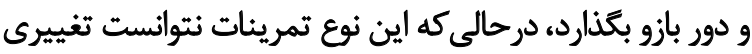

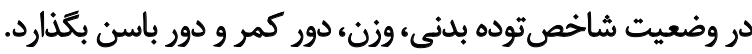

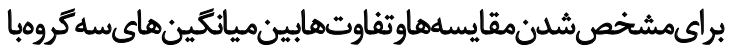

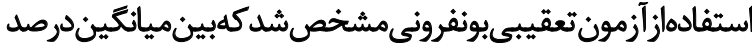

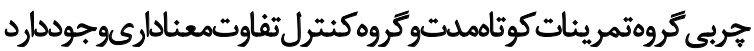

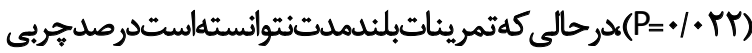

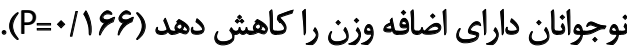

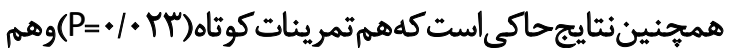

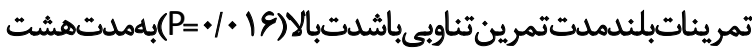

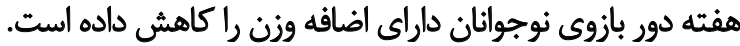
درنهايت استفاده از آزمون تعقيبى بونفرونى مشخص كرون كرد

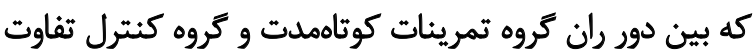

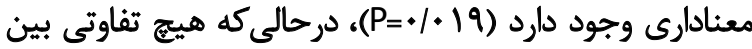

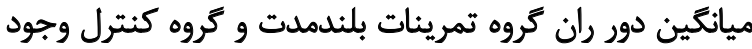

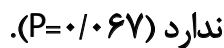

همجينين نتايج نشان داد هشت هفته تمرين كوتاه و بلندمدت

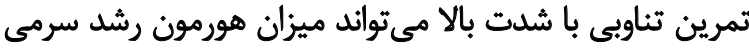

ديل و كاستيل ^استفاده شد. براى اندازهيرى GH أز روش الايزا

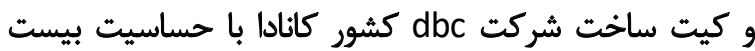

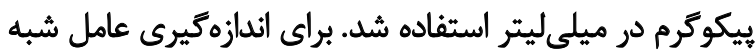

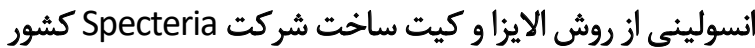
فنلاند با حساسيت هآ/ها إيكو كرم در ميلى ليتر استفاده شد. درصد ضريب تغييرات درون آزمونى در مورد همه آزمونهاي

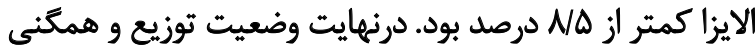

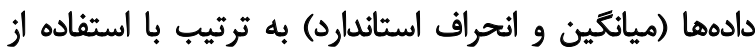

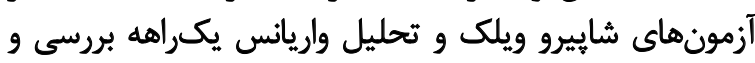

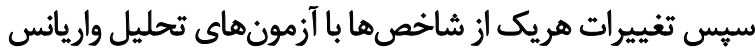

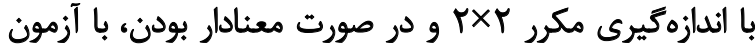

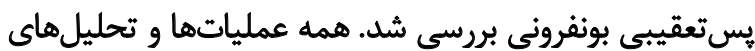

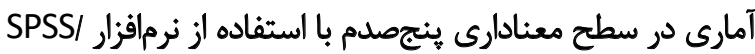
PASW تحت ويندوز نسخه ب ب انجام شد. ميانكين و انحراف استاندارد براى شاخصهاي سن، قد و مقادير

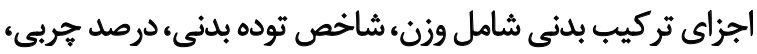

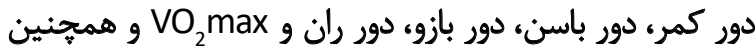

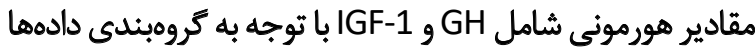

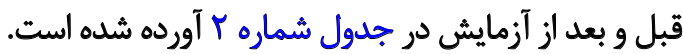

يس از بررسى مقادير ييش آزمون توسط آزمون شاييرو ويلك

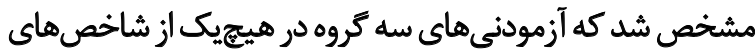

8. Dill \& Costill 
جدول شماره r. مثادير اجزاي تركيب بدنى و هورمونى قبل از شروع آزمايش در كروههاى مختلف

\begin{tabular}{|c|c|c|c|c|c|}
\hline \multirow{2}{*}{ سطح معنادارى آزمون } & \multicolumn{3}{|c|}{ ميانكين \$انحراف استاندارد } & \multirow{2}{*}{\multicolumn{2}{|c|}{ شاخص / ميروها }} \\
\hline & كتيرل & HIIT بلندمدت HIT & HIIT كوتاهمدت H & & \\
\hline- & $\mid \varphi / m \Psi \pm \pm(\Delta A$ & IF/MEI/Ye & $\mid \varphi / m \Psi \pm 1 / 80$ & & سن سن \\
\hline- & $\mid \Delta \% / M \pm I$ & $\mid Q F / I Y \pm A$ & $101 / 41 \pm 9$ & & \\
\hline ( & 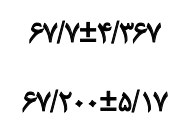 & 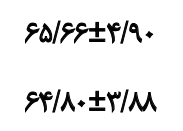 & 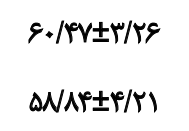 & يسيش أز ئهون & وزن (kg) \\
\hline .1 .98 & $\begin{array}{l}r R / L+ \pm r / M e \\
r V / I r \pm r / R e\end{array}$ & $\begin{array}{l}r Y / \Delta \Delta \pm Y / \bullet \varphi \\
r \Delta / Y Y \pm T / \cdot .\end{array}$ & $\begin{array}{l}T \& / \Delta \Lambda \pm Y / T Y \\
T Y / T E \pm V / \Delta P\end{array}$ & يسيشآزآزمون & درصد هربى \\
\hline.$/ 915$ & $\begin{array}{l}r R / T+ \pm T / M \\
r \& / \&+ \pm I / T\end{array}$ & $\begin{array}{l}\text { re/NTI/Rq } \\
\text { re/RA }\end{array}$ & 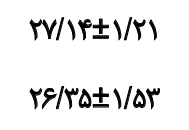 & يسيش أزئمون & $\mathrm{BMI}(\mathrm{kg} / \mathrm{m} 2)$ \\
\hline . IAAT & $\begin{array}{l}\text { V.FY } \\
1 /+49\end{array}$ & $\begin{array}{l}V / 4 q \\
V+r q\end{array}$ & $\begin{array}{l}1 /+r e \\
1 /+r\end{array}$ & يسيشآزئهون & نسبت دور كمر به باسن \\
\hline l.Va & 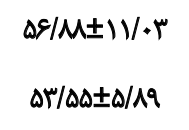 & 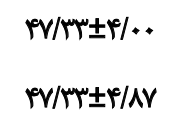 & $\begin{array}{l}P V / M \pm \Delta / \Delta V^{*} \\
P \Delta / Q q^{ \pm} \pm \Delta / T^{\prime} A\end{array}$ & يبش آزئهون & دور ران (cm) \\
\hline .1 .98 & $\begin{array}{l}r q / \Lambda I \pm r / \Delta \Delta \\
r \backslash / P P \pm r / r \varphi\end{array}$ & $\begin{array}{l}Y N M \pm Y \% I^{*} \\
r \Delta / V Q \pm T / V \Delta\end{array}$ & $\begin{array}{l}r V / W \pm I / A A^{*} \\
r \Delta / / Y \pm T / R Y\end{array}$ & يَيش آز يسمون & دور بازو (cm) \\
\hline ./WE & $\begin{array}{l}r V / \& \Delta \pm r / r q q \\
r r / \cdot N \pm r / / R\end{array}$ & $\begin{array}{l}r T / N \pm(/ \Delta)^{*} \\
r E / V \cdot \pm 1 / 99\end{array}$ & 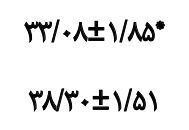 & يسئ الأزمون & $\begin{array}{c}\mathrm{Vo}_{2} \max \\
(\mathrm{ml} / \mathrm{kg} / \mathrm{min})\end{array}$ \\
\hline . & $\begin{array}{l}. / r A \pm+1+\infty \\
+/ r A \pm+1+F\end{array}$ & $\begin{array}{l}. / R E \pm+1 \cdot r \\
. / 4 t+\%\end{array}$ & $\begin{array}{l}\cdot / r+ \pm \cdot / \cdot r \\
\cdot / r \Delta \pm+/+r *\end{array}$ & يسيشآزيمون & هورمون رشد (ر/l) \\
\hline$+1+1 \mid$ & $\begin{array}{l}\text { IENFFEI./FT } \\
\text { IENADEVAQ }\end{array}$ & $\begin{array}{l}1 \Delta Q / Y T \pm 1+1+0 \\
18 Y / M \pm V / \Delta q\end{array}$ & 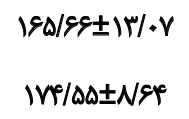 & يسيش آلزئون & IGF-1 ( $\mu \mathrm{g} / \mathrm{l})$ \\
\hline
\end{tabular}

نوجوانان داراى اضافه وزن را افزايش داد. همسو با تحقيق حاضر،

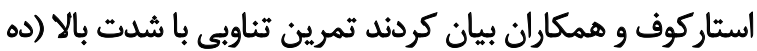

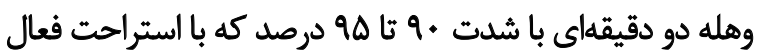

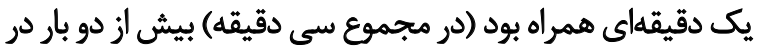

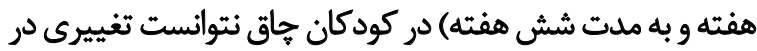

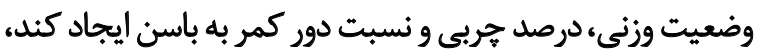

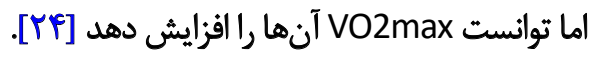
از آنجا كه مقادير VO

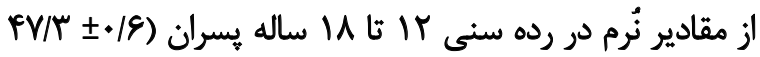

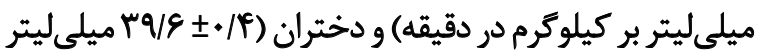

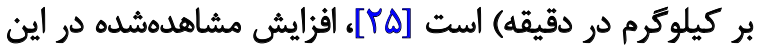

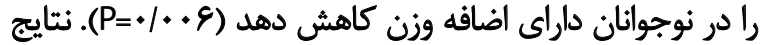

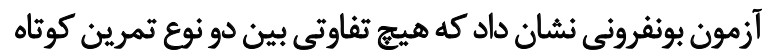

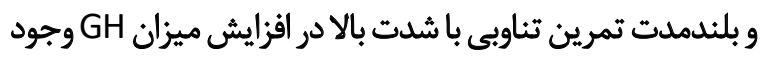

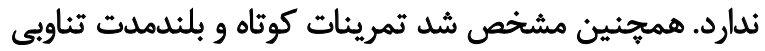

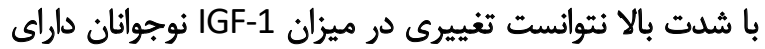

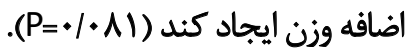
$\stackrel{\leftrightarrow}{\leftrightarrow}$ مطالعه حاضر نشان داد كه هشت هفته تمرين تناوبى با شدت

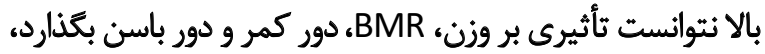

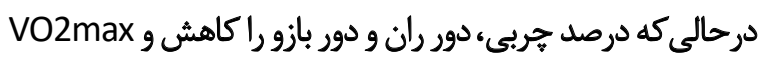


درصد حداكثر اكسيرن مصرفى) در نوجوانان هاق بيان كردند

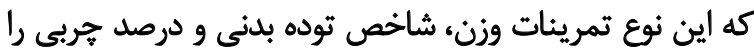

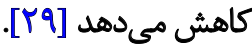

در مطالعه ديكرى، دى آراخو و همكاران بيان كردند تمرين

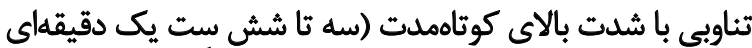

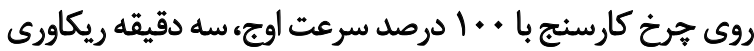

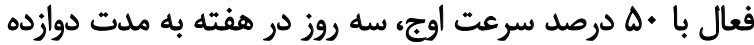

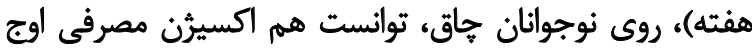

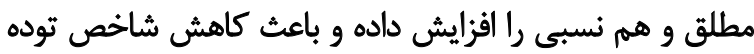

بدنى و بهبود ساير شاخص هاى تركيب بدنى شود [ـ"r].

كونك و همكاران نيز در مطالعهاي كه روى دختران جوان جوان

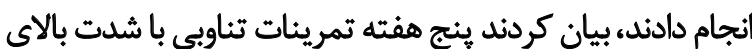

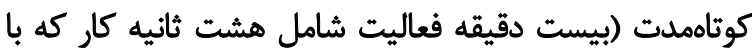

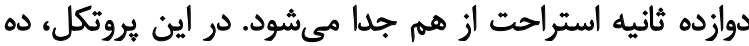

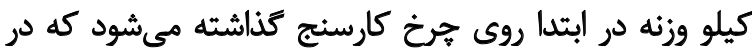

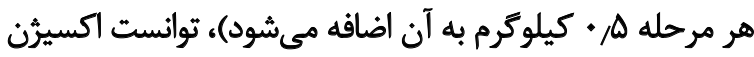

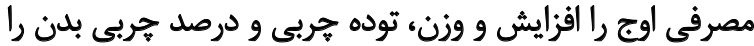

كاهش دهد [آن]

در تحقيق رانتً و همكاران، دوازده هفته تمرين تناوبى با

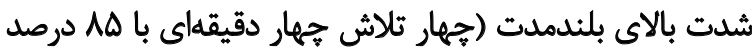

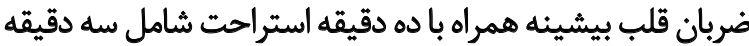

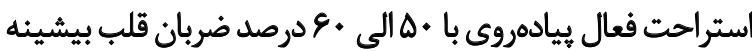

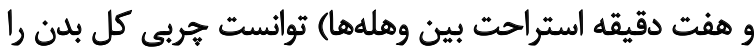

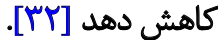

از سوى ديخر، قلىزاده و همكاران، تفاوت تأثير دو نوع تمرين

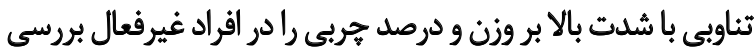

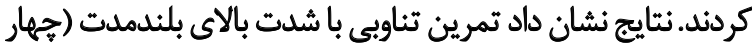

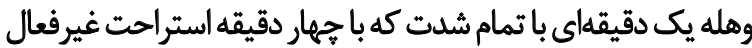

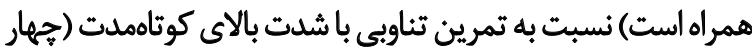

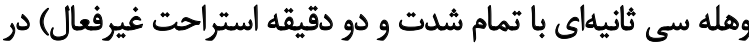

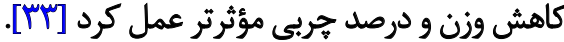
در مطالعهاى ديكر، تانگ و همكاران با بررسى اثر دوازده هفته

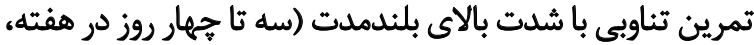

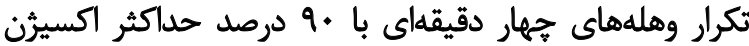

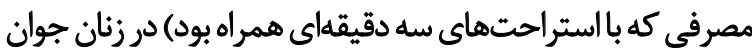

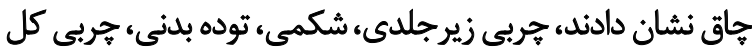

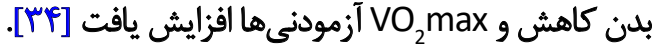

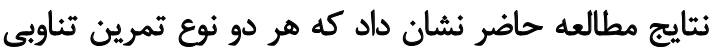

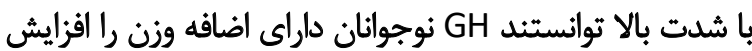

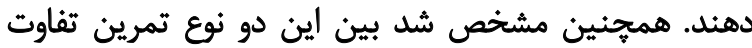

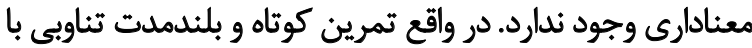

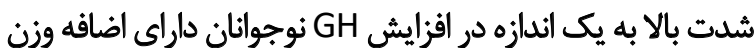

مطالعه مي تواند مزاياي سلامت بيشمارى به ارمغان بياورد. در مطالعهاى ديگر، مورفى و همكاران نيز بيان كردند جهار تهار

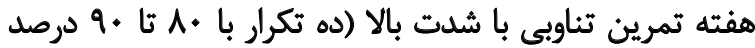

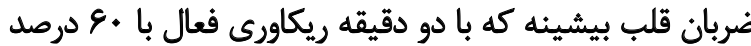

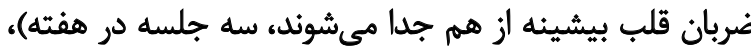

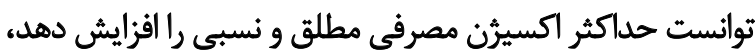

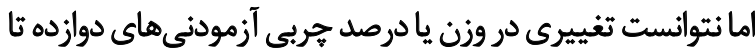

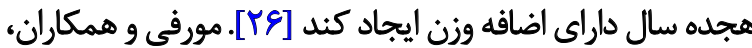

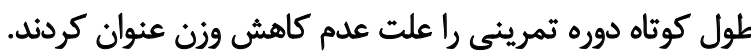

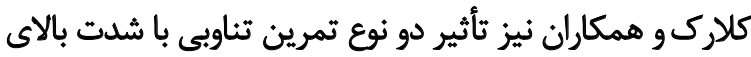

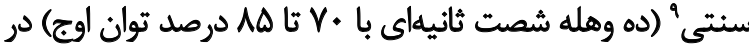

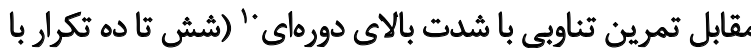

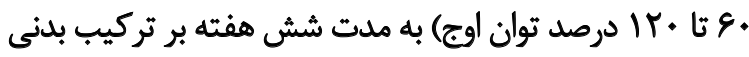

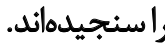

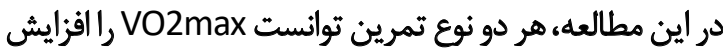

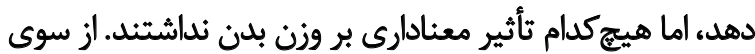

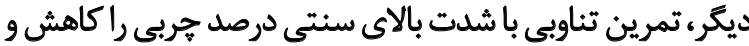

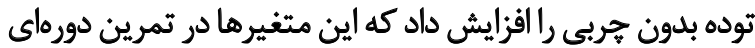

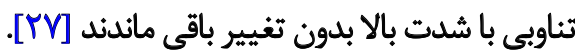
مكانيسمهاى دقيق افزايش VO2max در اين مطالعه ناشناخته است، اما يك توضيح احتمالى، تقويت محتوا يا ياعملكرد

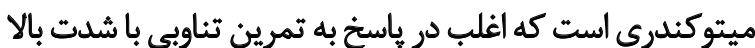

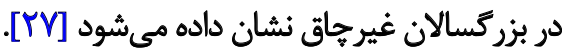
اما ناهمسو با نثايج مطالعه حاضر، راسيل و همكاران بيان كردئد

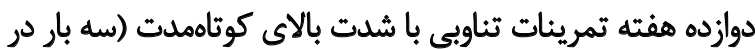

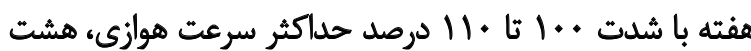

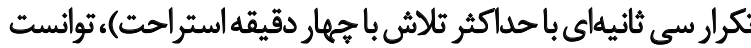

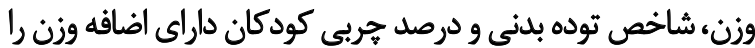

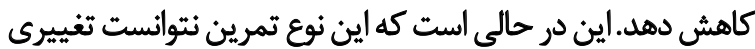

در وضعيت VO اتر جه مطالعه حاضر نتوانست كاهش معنادارى در وزن و

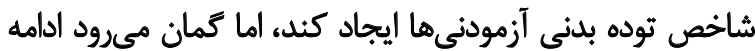

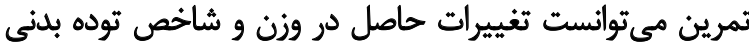

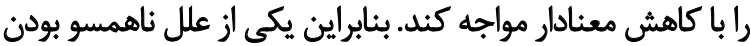

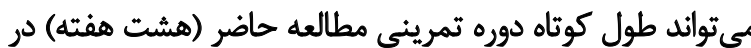

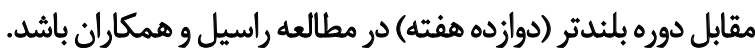
همجنين لازر و همكاران با بررسى اثر شش هفته تمرين تناوبى

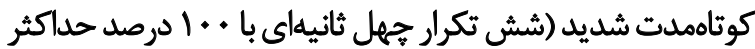

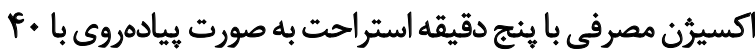

9. Traditional

10. Periodized 


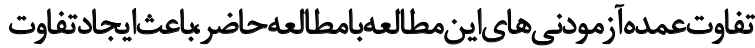

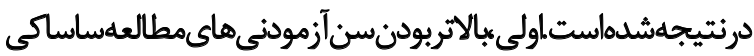

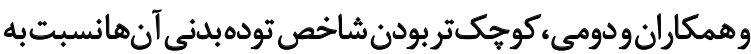

مطالعه حاضر است [19].

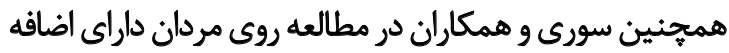

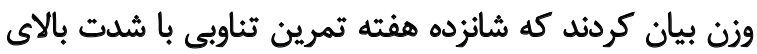

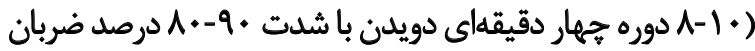

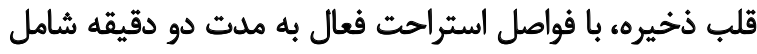

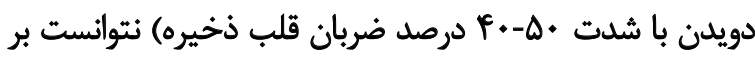

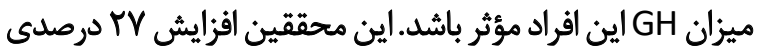

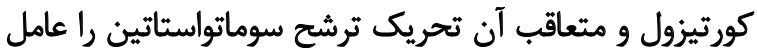

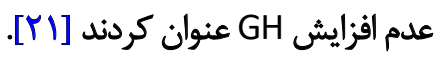

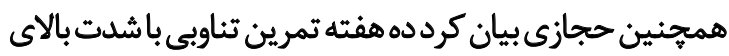

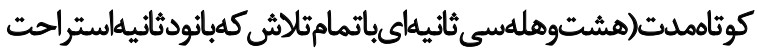

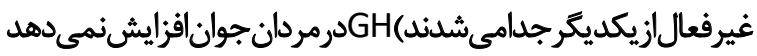

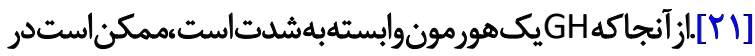
طول زمان به سبب سازكارى بدون تغيير شود.

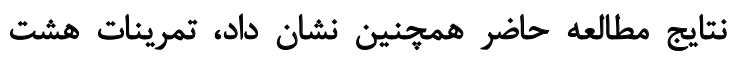

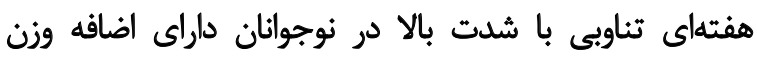

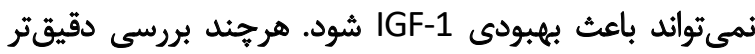

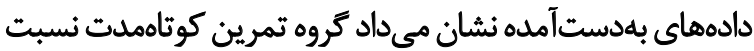

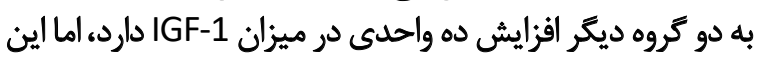
افزايش از لحاظ آمارى معنادار نبود.

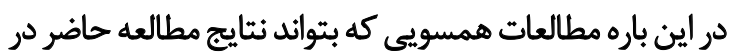

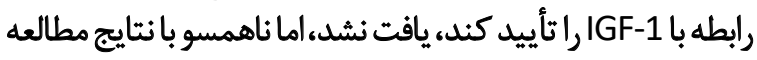

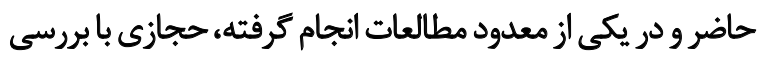

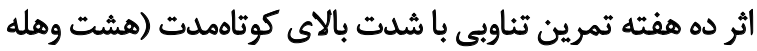

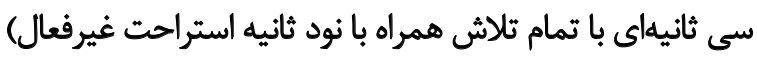

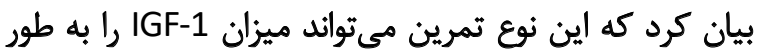

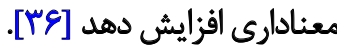

از آنجا كه نوع تمرين در مطالعه حجازى كوتاهمدت است،

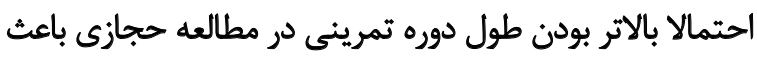

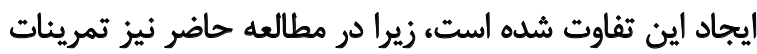

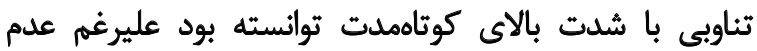

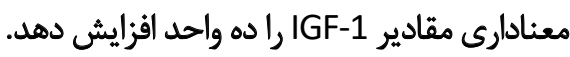

در ديكر مطالعه انجامشده، هربرت و همكاران بيان كردند

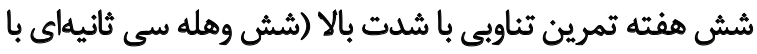

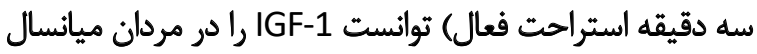

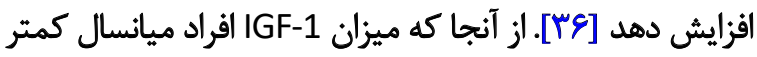

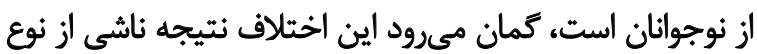

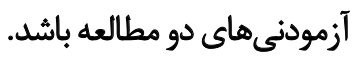

مؤثر هستند. تمرينات بى هوازى با شدت بالا معمولاً به افزايش

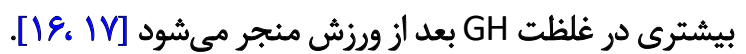
همسو با نتايج مطالعه حاضر، عبدالرحمان و همكاران كزارش

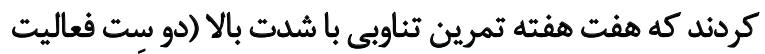

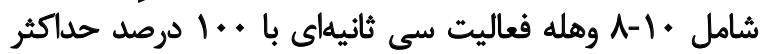

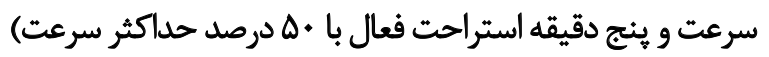

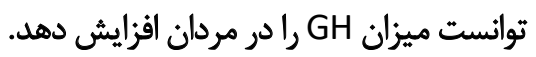

اين محققين يكى از علل افزايش GH به واسطه تمرينات

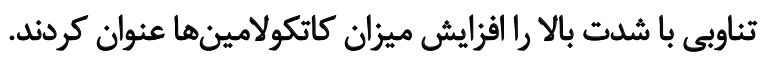

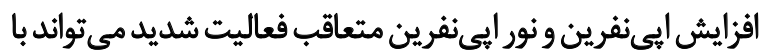

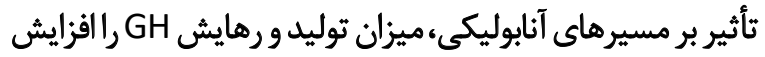

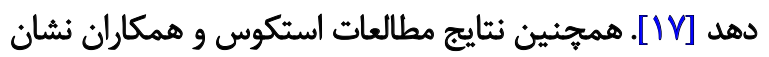

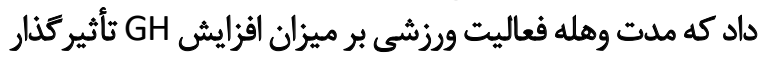

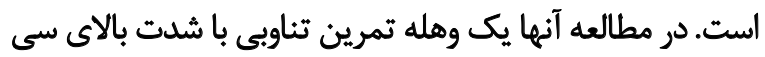

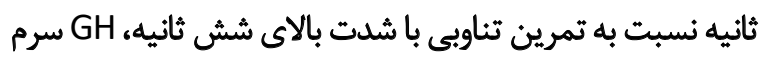
را بيشتر افزايش داد.

محققان علت اين تفاوت را بالا بودن ميزان كار انجامشده در

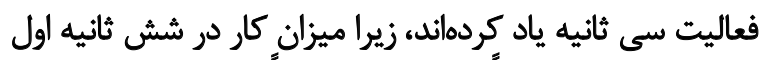

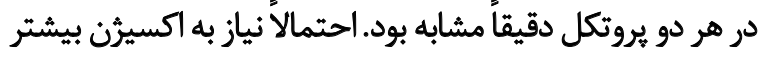

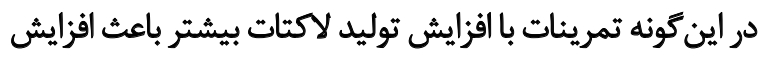

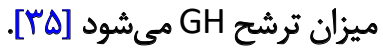

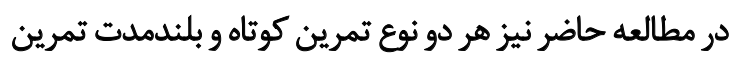

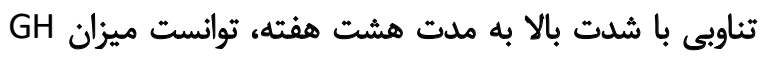

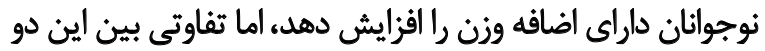

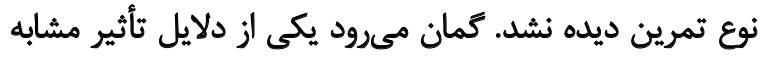

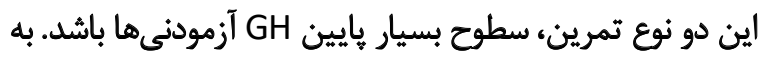

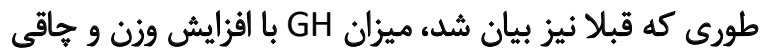

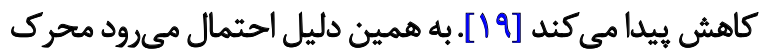

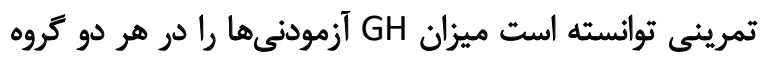

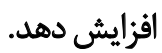

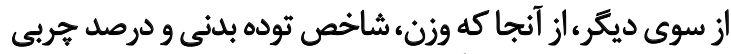

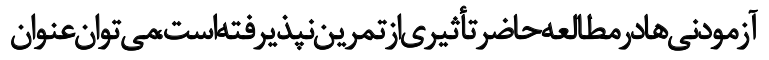

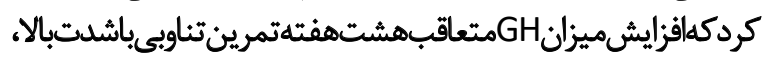

$$
\text { مستقل از وضعيت تركيب بدنى است. }
$$

اما برخلاف نتايج مطالعه حاضر، ساساكى و همكاران بيان

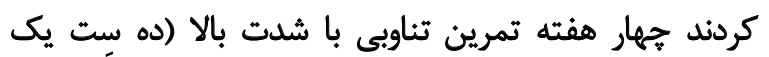

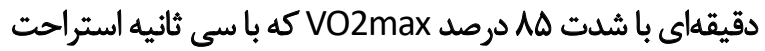
تاز يكديكر جدا مى شوند) نتوانست بر ميزان GH مردان غيرفي تأثيرى بكذارد.

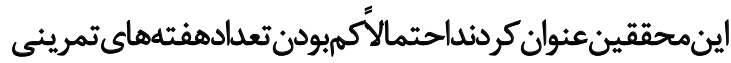

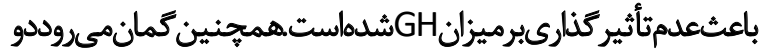


اثرات انواع تمرينات ورزشي به طور بالقوه بر وضعيت سلامت

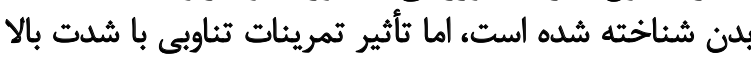

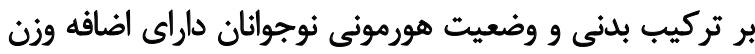

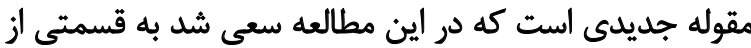

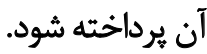

$$
\text { تنيجهيرى }
$$

اتر جه تمرين تناوبى با شدت بالا در اين مطالعه نتوانست

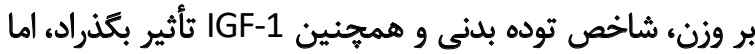

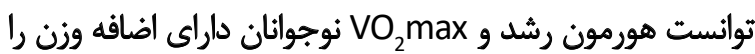

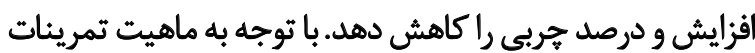

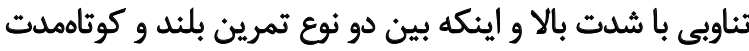

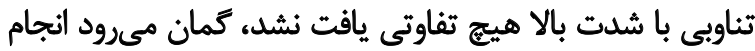

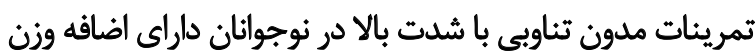
بتواند مزاياى سلامت را براي اين عزيزان بها ارمغان بيان بياورد

مالاحظات اخلاقى

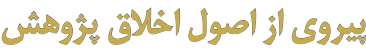

IR.TABRIZU. اين مطالعه داراى كد اخلاق به شماره REC.1398.021 از دانشكاه تبريز است.

$$
\text { tho }
$$

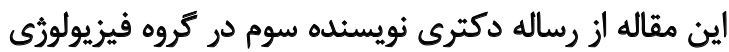
ورزشى دانشكده تربيت بدنى و علوم ورزشى دانش دانشكاه تبريز استخراج شده است.

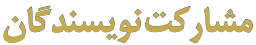

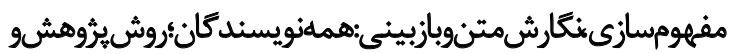

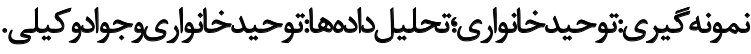

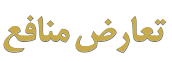

در اين مقاله هيجگونه تعارض منافعى وجود ندارد. 


\section{Reference}

[1] Sahoo K, Sahoo B, Choudhury AK, Sofi NY, Kumar R, Bhadoria AS. Childhood obesity: Causes and consequences. J Family Med Prim Care. 2015; 4(2):187-92. [DOI:10.4103/2249-4863.154628] [PMID] [PMCID]

[2] Pozza C, Isidori AM. What's behind the obesity epidemic. In: Laghi A Rengo M, editors. Imaging in bariatric surgery. Germany: Springer; 2018. [DOI:10.1007/978-3-319-49299-5_1]

[3] World Health O. Noncommunicable diseases: Risk factors [Internet]. 2021 [Updated 2021]. Available from: https://www.who.int/data/gho/ data/themes/topics/topic-details/GHO/ncd-risk-factors

[4] Makimura H, Stanley T, Mun D, You SM, Grinspoon S. The effects of central adiposity on Growth Hormone (GH) response to GH-releasing hormone-arginine stimulation testing in men. J Clin Endocrinol Metab. 2008; 93(11):4254-60. [DOI:10.1210/jc.2008-1333] [PMID] [PMCID]

[5] Stanley TL, Feldpausch MN, Murphy CA, Grinspoon SK, Makimura H. Discordance of IGF-1 and GH stimulation testing for altered GH secretion in obesity. Growth Horm IGF Res. 2014; 24(1):10-5. [DOI:10.1016/j. ghir.2013.11.001] [PMID] [PMCID]

[6] Henry B, Jensen EA, List EO, Berryman DE. Obesity and the Growth Hormone Axis. In: Nillni EA, editor. Textbook of energy balance, neuropeptide hormones, and neuroendocrine function. Germany: Springer; 2018. [DOI:10.1007/978-3-319-89506-2_12]

[7] Lim HK, Dong JY, Bae SM, Kwon SC, inventors; Hanmi Pharmaceutical Co Ltd, assignee. A novel liquid formulation of long-acting human growth hormone conjugate. United States patent application US 16/066,877 [Internet]. 2019 [Updated 2019 Jan 24]. Available from: https://patents. google.com/patent/US20190022183A1/en

[8] Bartke A. Growth hormone and aging: Updated review. World J Mens Health. 2019; 37(1):19-30. [DOI:10.5534/wjmh.180018] [PMID] [PMCID]

[9] Higashi Y, Gautam S, Delafontaine P, Sukhanov S. IGF-1 and cardiovascular disease. Growth Horm IGF Res. 2019; 45:6-16. [DOI:10.1016/j. ghir.2019.01.002] [PMID] [PMCID]

[10] Homaee H, Moradi L, Azarbayjani M, Peeri M. Effect of high intensity exercise training (HIIT) and endurance training on weight loss and C-reactive protein in obese men. Int J Biosci. 2014; 4(9):190-6. [DOI:10.12692/ijb/4.9.190-196]

[11] Gillen JB, Gibala MJ. Is high-intensity interval training a time-efficient exercise strategy to improve health and fitness? Appl Physiol Nutr Metab. 2013; 39(3):409-12. [DOI:10.1139/apnm-2013-0187] [PMID]

[12] Gibala MJ, Little JP, Van Essen M, Wilkin GP, Burgomaster KA, Safdar $A$, et al. Short-term sprint interval versus traditional endurance training: Similar initial adaptations in human skeletal muscle and exercise performance. J Physiol. 2006; 575(Pt 3):901-11. [DOI:10.1113/jphysiol.2006.112094] [PMID] [PMCID]

[13] Heydari M, Freund J, Boutcher SH. The effect of high-intensity intermittent exercise on body composition of overweight young males. J Obes. 2012; 2012:480467. [DOI:10.1155/2012/480467] [PMID] [PMCID]

[14] Mangine GT, Hoffman JR, Gonzalez AM, Townsend JR, Wells AJ, Jajtner $A R$, et al. Exercise-induced hormone elevations are related to muscle growth. J Strength Cond Res. 2017; 31(1):45-53. [DOI:10.1519/ JSC.0000000000001491] [PMID]

[15] Sylta o, Sandbakk o, Hammarstom D, Danielsen J, Skovereng K, Ronestad $B$, et al. Effects of high-intensity training on physiological and hormonal adaptions in well-trained cyclists. Med Sci Sports Exerc. 2017; 49(6):1137-46. [DOI:10.1249/MSS.0000000000001214] [PMID]
[16] Collier SR, Collins E, Kanaley JA. Oral arginine attenuates the growth hormone response to resistance exercise. J Appl Physiol. 2006; 101(3):848-52. [DOI:10.1152/japplphysiol.00285.2006] [PMID]

[17] Stokes KA. Nevill ME, Hall GM, and Lakomy HKA. Growth hormone responses to repeated maximal cycle ergometer exercise at different pedaling rates. J Appl Physiol. 2002; 92(2):602-8. [DOI:10.1152/jappl.2002.92.2.602] [PMID]

[18] Abderrahman AB, Rhibi F, Ouerghi N, Hackney AC, Saeidi A, Zouhal $\mathrm{H}$. Effects of recovery mode during high intensity interval training on glucoregulatory hormones and glucose metabolism in response to maximal exercise. J Athl Enhanc. 2018; 7(3):292. [DOI:10.4172/23249080.1000292] [PMID] [PMCID]

[19] Sasaki H, Morishima T, Hasegawa Y, Mori A, ljichi T, Kurihara T, et al. 4 weeks of high-intensity interval training does not alter the exercise-induced growth hormone response in sedentary men. Springerplus. 2014; 3:336. [DOI:10.1186/2193-1801-3-336] [PMID] [PMCID]

[20] Soori R, Rezaeian N, Salehyian O. [Effects of interval training on leptin and hormone levels affecting lipid metabolism in young obese/ overweight men (Persian)]. Iran J Endocrinol Metab. 2012; 14(3):248-56. http://ijem.sbmu.ac.ir/article-1-1234-fa.html

[21] Hejazi SM. Effects of High Intensity Interval Training on Plasma Levels of GH and IGF-I. IntI J Med Res Health Sci. 2017; 6(4):55-9. https://www. indianjournals.com/ijor.aspx?target=ijor:ijmrhs\&volume=6\&issue $=4 \& a$ rticle $=009$

[22] Agha-Alinejad H, Gharakhanlou R, Farzad B, Bayati M. [Norms of anthropometric, body composition measures and prevalence of overweight and obesity in urban populations of Iran (Persian)]. J Shahrekord Univ Med Sci. 2014; 15(6):18-27. http://journal.skums.ac.ir/browse. php?a_code=A-10-765-1\&slc_lang=en \&sid=1

[23] Thivel D, Masurier J, Baquet G, Timmons BW, Pereira B, Berthoin S, et al. High-intensity interval training in overweight and obese children and adolescents: Systematic review and meta-analysis. J Sports Med Phys Fitness. 2018; 59(2):310-24. [DOI:10.23736/S0022-4707.18.08075-1] [PMID]

[24] Starkoff BE, Eneli IU, Bonny AE, Hoffman RP, Devor ST. Estimated aerobic capacity changes in adolescents with obesity following high intensity interval exercise. Int J Kinesiol Sports Sci. 2014; 2(3):1-8. [DOI:10.7575/ aiac.ijkss.v.2n.3p.1]

[25] Welk GJ, Laurson KR, Eisenmann JC, Cureton KJ. Development of youth aerobic-capacity standards using receiver operating characteristic curves. Am J Prev Med. 2011; 41(4 S 2):S111-6. [DOI:10.1016/j.amepre.2011.07.007] [PMID]

[26] Murphy A, Kist C, Gier AJ, Edwards NM, Gao Z, Siegel RM. The feasibility of high-intensity interval exercise in obese adolescents. Clin Pediatr. 2015; 54(1):87-90. [DOI:10.1177/0009922814528038] [PMID] [PMCID]

[27] Clark A, Annie B, DeRevere JL, Astorino TA. Effects of various interval training regimes on changes in maximal oxygen uptake, body composition, and muscular strength in sedentary women with obesity. Eur J Appl Physiol. 2019; 119(4):879-88. [DOI:10.1007/s00421-019-04077-x] [PMID]

[28] Racil G, Ounis OB, Hammouda O, Kallel A, Zouhal H, Chamari K, et al. Effects of high vs. moderate exercise intensity during interval training on lipids and adiponectin levels in obese young females. Eur J Appl Physiol. 2013; 113(10):2531-40. [DOI:10.1007/s00421-013-2689-5] [PMID]

[29] Lazzer S, Tringali G, Caccavale M, De Micheli R, Abbruzzese L, Sartorio A. Effects of high-intensity interval training on physical capacities and substrate oxidation rate in obese adolescents. J Endocrinol Invest. 2017; 40(2):217-26. [DOI:10.1007/s40618-016-0551-4] [PMID] 
[30] De Araujo ACC, Roschel H, Picanco AR, do Prado DML, Villares SMF, de sa Pinto $A$, et al. Similar health benefits of endurance and high-intensity interval training in obese children. PloS one. 2012; 7(8):e42747. [DOI:10.1371/journal.pone.0042747] [PMID] [PMCID]

[31] Kong Z, Fan X, Sun S, Song L, Shi Q, Nie J. Comparison of high-intensity interval training and moderate-to-vigorous continuous training for cardiometabolic health and exercise enjoyment in obese. PLoS One. 2016; 11(7):e0158589. [DOI:10.1371/journal.pone.0158589]

[32] Zhang H, K Tong T, Qiu W, Wang J, Nie J, He Y. Effect of high-intensity interval training protocol on abdominal fat reduction in overweight Chinese women: A randomized controlled trial. Kinesiol. 2015; 47(1):57-66. https://hrcak.srce.hr/140252

[33] Gholizadeh, Kurdi, Akbarnezhad, Afusi Gh, Mohammadi S. [The effect of two types of very intense intermittent exercise (HIIT) on fat and carbohydrate oxidation in overweight young men (Persian)]. J Sport Biol Sci. 2017; 9(2):157-69. [DOI:10.22059/JSB.2017.41874.659]

[34] Tong TK, Zhang H, Shi H, Liu Y, Ai J, Nie J, et al. Comparing time efficiency of sprint vs. high-intensity interval training in reducing abdominal visceral fat in obese young women: A randomized, controlled trial. Front Physiol. 2018; 9:1048. [DOl:10.3389/fphys.2018.01048] [PMID] [PMCID]

[35] Utz AL, Yamamoto A, Hemphill L, Miller KK. Growth hormone deficiency by growth hormone releasing hormone-arginine testing criteria predicts increased cardiovascular risk markers in normal young overweight and obese women. J Clin Endocrinol Metab. 2008; 93(7):2507-14. [DOI:10.1210/jc.2008-0169] [PMID] [PMCID]

[36] Herbert P, Hayes LD, Sculthorpe N, Grace FM. High-intensity interval training (HIIT) increases insulin-like growth factor-I (IGF-I) in sedentary aging men but not masters' athletes: An observational study. Aging Male. 2017; 20(1):54-9. [DOI:10.1080/13685538.2016.1260108] [PMID] 
This Page Intentionally Left Blank 